\title{
Technical considerations when using the EEG export of the SEDLine Root device
}

\author{
Falk von Dincklage ${ }^{1} \cdot$ Carlo Jurth $^{1} \cdot$ Gerhard Schneider $^{2} \cdot$ Paul S García $^{3} \cdot$ Matthias Kreuzer $^{2} \mathbb{0}$
}

Received: 19 February 2020 / Accepted: 10 August 2020 / Published online: 19 August 2020

(c) The Author(s) 2020

\begin{abstract}
Electroencephalographic (EEG) patient monitoring during general anesthesia can help to assess the real-time neurophysiology of unconscious states. Some monitoring systems like the SEDLine Root allow export of the EEG to be used for retrospective analysis. We show that changes made to the SEDLine display during recording affected the recorded EEG. These changes can strongly impact retrospective analysis of EEG signals. Real-time changes of the feed speed in the SEDLine Root device display modifies the sampling rate of the exported EEG. We used a patient as well as a simulated EEG recording to highlight the effects of the display settings on the extracted EEG. Therefore, we changed EEG feed and amplitude resolution on the display in a systematic manner. To visualize the effects of these changes, we present raw EEG segments and the density spectral array of the recording. Changing the display's amplitude resolution affects the amplitudes. If the amplitude resolution is too fine, the exported EEG contains clipped amplitudes. If the resolution is too coarse, the EEG resolution becomes too low leading to a low-quality signal making frequency analysis impossible. The proportion of clipped or zero-line data caused by the amplitude setting was $>60 \%$ in our sedated patient. Changing the display settings results in undocumented changes in EEG amplitude, sampling rate, and signal quality. The occult nature of these changes could make the analysis of data sets difficult if not invalid. We strongly suggest researchers adequately define and keep the EEG display settings to export good quality EEG and to ensure comparability among patients.
\end{abstract}

Keywords Monitoring $\cdot$ Electroencephalography $\cdot$ Anesthesia general $\cdot$ Patients

\section{Introduction}

Monitoring the brain during general anesthesia by means of electroencephalographic (EEG) recordings can help to assess an unconscious patient, as anesthetic drugs induce

Electronic supplementary material The online version of this article (https://doi.org/10.1007/s10877-020-00578-9) contains supplementary material, which is available to authorized users.

Matthias Kreuzer

m.kreuzer@tum.de

1 Klinik für Anästhesiologie m.S. operative Intensivmedizin (CCM/CVK), Charité - Universitätsmedizin Berlin, Berlin, Germany

2 Department of Anesthesiology and Intensive Care, Klinikum rechts der Isar, School of Medicine, Technical University of Munich, Munich, Germany

3 Department of Anesthesiology, Neuroanesthesia Division, Columbia University Medical Center, New York Presbyterian Hospital, New York, USA changes in neuronal firing that are reflected as specific EEG activity. Most commonly the unconscious brain exhibits a general slowing of the EEG oscillations and an increase in amplitude [1]. Many commercial devices use EEG spectral information to produce a dimensionless index that represents the hypnotic component of surgical anesthesia. The SEDLine Root device, is a brain function monitor (Masimo, Irvine, CA, USA) [2]. It processes frontal EEG information to calculate the patient state index (PSI) that attempts to mark the patient's level of arousal. The device also has an automated method for burst suppression detection. The device provides an export function that allows data collection and retrospective analysis of the recorded EEG. The data can be stored in the European data file format (EDF) [3] on an USB stick. Possession of these EEG recordings in an easily readable format provides the opportunity to conduct research on EEG features that occur during general anesthesia. Earlier versions of the SEDline monitor were used to detect burst suppression EEG and describe alpha oscillatory activity as a target for anesthesia maintenance [4]. In this 
manuscript, we describe how intraoperative modification of the display features can not only influence the displayed EEG but also the recorded EEG file when stored in the EDF file format. These difficulties include undocumented changes in EEG sampling rate and amplitude as well as data "clipping" (EEG amplitudes beyond the y-axis limits). These changes can lead to corrupted EEG files and perhaps falsify the results from subsequent analysis.

\section{Methods}

For our investigation we used EEG recordings obtained from a sedated intensive care patient that was included in the clinical trial "Validation of methods for monitoring nociception and pain prediction in the ICU". This study was performed in accordance with the ethical standards as laid down in the 1964 Declaration of Helsinki and its later amendments. It was approved and the requirement for written informed consent was waived by the Institutional Research Ethics Committee of Charité - Universitätsmedizin Berlin (vote number: EA1/151/16). The trial was registered prior to patient enrollment at the German Clinical Trial Register (DRKS00011206; Principal investigator: Dr. Falk von Dincklage, Date of registration: 11.01.2017).

\subsection{SEDLine EEG recording}

When using the SEDLine, an electrode sensor is attached to the patient's forehead. The sensor consists of six electrodes that record brain electrical activity from recording positions Fp1, Fp2, F7 and F8 according to the international 10-20 system with the reference and ground electrode placed on the center of the forehead (around Fz). The SEDLine monitor processes the EEG information to calculate the PSI. It also displays the changes in spectral EEG power over time as a density spectral array (DSA) on the monitor's display to provide the anesthesiologist with additional information regarding the oscillatory composition of the EEG. Further, the raw EEG can also be displayed on the monitor. In order to optimally visualize the EEG trace, the anesthesiologist can adjust the time-scale of the EEG as well as the amplitude resolution. The scaling is either $15 \mathrm{~mm}$ or $30 \mathrm{~mm}$ per second and the amplitudes can be adjusted to 1, 2, 3, 5, 10, 25, 50, or $100 \mu \mathrm{V}$ per $\mathrm{mm}$.

\subsection{SEDLine EEG export}

It is possible to export a recorded EEG file from the SEDLine Root device in European Data Format (EDF file, .edf extension).Using the export function, the EEG can be exported to a USB-drive as an EDF file.
The EDF files consist of a header record and a data record. The data record contains the time series data, i.e., the amplitude values recorded at equally-spaced time points. In exported SEDLine EEG files the data record contains numeric information from the four frontal EEG channels. The header record contains information regarding electrode positions, patient and time information etc. The sample rate is not given explicitly, but can be obtained by dividing the value of the header variable 'samples' by the value of the header variable 'duration'. Hence, one sample rate value can be derived that reflects the sample rate for the entire recording. The sample rate is defined at the beginning of the recording.

\subsection{Change of EEG display protocol}

\subsubsection{Clinical EEG recording}

We suspected that the choice of parameter settings on the SEDLine display during EEG recording may influence the raw EEG that can be exported to the EDF file. Thus, we investigated this possible effect using EEG recordings obtained from a sedated intensive care patient. The patient used for EEG measurement presented the following characteristic: male, 55 years of age, light analgosedation using propofol, clonidine as well as methyl-lorazepam in the postoperative setting. Because we were only interested in the question of how the monitor settings influence the exported EEG, one patient was considered sufficient. We decided on a patient under propofol-induced sedation because (i) the EEG is consistent during constant low/moderate infusion doses, (ii) propofol causes prominent EEG alpha band activity [5], and (iii) there is no EEG contamination by surgical artifacts in this post-operative patient.

We started the EEG recording with the $30 \mathrm{~mm} / \mathrm{s}$ timescale. We then changed the time-scale to $15 \mathrm{~mm} / \mathrm{s}$ and started our stepwise protocol with an initial amplitude setting of $1 \mu \mathrm{V} / \mathrm{mm}$. We changed the amplitude resolution, approximately every two minutes, in a stepwise fashion $(\mu \mathrm{V} /$ mm in eight steps $; \rightarrow 2 \rightarrow 3 \rightarrow 5 \rightarrow 10 \rightarrow 25 \rightarrow 50 \rightarrow 100$ ), while keeping the feed constant at $15 \mathrm{~mm} / \mathrm{s}$. After completing the eight steps we changed the feed to $30 \mathrm{~mm} / \mathrm{s}$ and reversed the amplitude setting from $100 \mu \mathrm{V} / \mathrm{mm}$ to $1 \mu \mathrm{V} / \mathrm{mm}$ in the stepwise fashion described before for another eight steps.

This stepwise protocol is also presented in Fig. 1. We then exported this recording as an EDF file and analyzed the EEG with MATLAB R2017b (The MathWorks, Natick, MA, USA). We based our analyses on the sample rate indicated in the header of the EDF file, which in our case was $178 \mathrm{~Hz}$, because we started with the $30 \mathrm{~mm} / \mathrm{s}$ feed. For the analyses, we used channel L1, i.e., the signal recorded from position Fp1. 


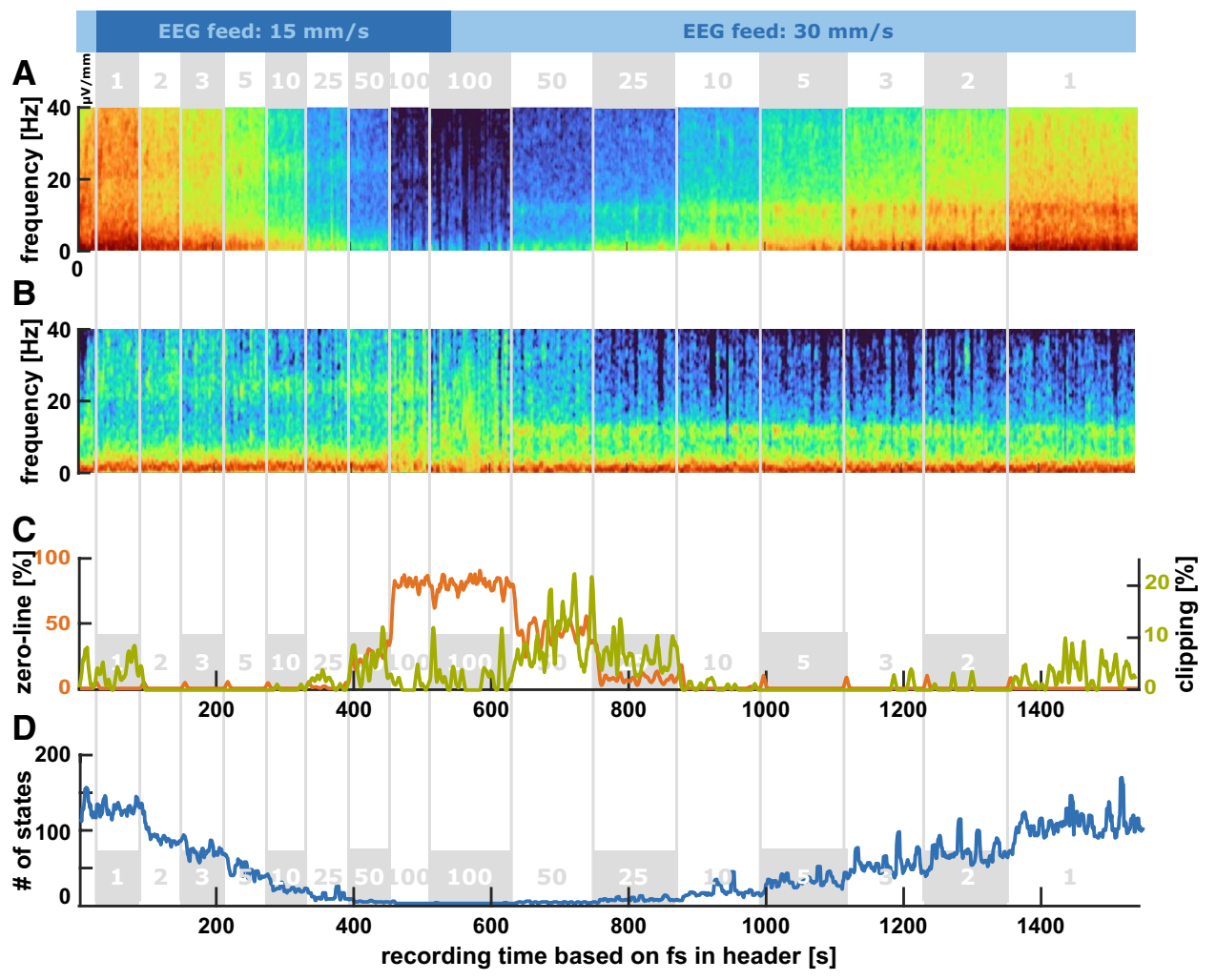

Fig. 1 Density spectral arrays (DSA) of the (relative) power spectral density (PSD) for the different display settings depicted above the DSA as well as the amount of clipping and zero-lines in these episodes a DSA of the spectral power: Depending on the amplitude setting the recorded EEG amplitudes, i.e., the colors in the DSA change. The width of the 'unchanged' settings relates to the EEG feed b DSA indicating the relative power: Using the relative power can correct for the change in EEG amplitude, but the influence of the EEG feed still remains c Amount of clipping (green) and zero-lines (orange):

\subsubsection{Simulated EEG recording}

We further replayed a simulated EEG trace to the SEDLine using an EEG player [6] to channel L1 (Fp1) and channel L2 (F7). Therefore, we generated a white noise signal using the MATLAB rand function and filtered the signal to the 9-11 $\mathrm{Hz}$ range using the MATLAB filtfilt function. We then conducted a stepwise replay of the signal starting with a $30 \mathrm{~mm} / \mathrm{s}$ feed (i.e., a $\mathrm{f}_{\mathrm{s}}=178 \mathrm{~Hz}$ ) and a $1 \mu \mathrm{V} / \mathrm{mm}$ amplitude resolution. We increased the amplitude setting every $30 \mathrm{~s}$, changed to a feed of $15 \mathrm{~mm} / \mathrm{s}$ after $30 \mathrm{~s}$ in the $100 \mu \mathrm{V} / \mathrm{mm}$ setting. Then we decreased the amplitude resolution again in the same manner. For the analyses, we used channel L1, i.e., the signal recorded from position Fp1. For the evaluation of clipping, we used the signals from Fp1 and F7 in order to evaluate differences. the amount of clipped EEG amplitudes is substantial for the $1 \mu \mathrm{V} /$ $\mathrm{mm}$ setting as well as for the 50 and $25 \mu \mathrm{V} / \mathrm{mm}$ setting during the $30 \mathrm{~mm} / \mathrm{s}$ feed. There the clipping is caused by the low resolution of the signal that also causes the high fraction of zero-lines during the $100 \mu \mathrm{V} / \mathrm{mm}$ setting. $\mathbf{d}$ The number of states, i.e., the number of different amplitude values in the EEG recording decreased with an increase of the EEG amplitude setting. (The trend was smoothed with a moving mean of 5 points)

\subsection{Analysis of the EEG stored in the EDF file}

In order to examine possible changes in the EEG recording induced by changes in the display settings we calculated the power spectral density (PSD) of the EEG for overlapping EEG segments of $5 \mathrm{~s}$ length with a shift of $1 \mathrm{~s}$. Therefore, we used the MATLAB pwelch function with default settings and a NFFT $=512$. We also calculated the normalized PSD (nPSD) by dividing the PSD for each $5 \mathrm{~s}$ EEG episode by the sum of power in the 0.4 to $30 \mathrm{~Hz}$ range. We also used a custom MATLAB function to evaluate the amount of clipping in the recorded EEG for each feed and amplitude display setting. Clipping is a signal distortion that limits measures of the EEG amplitude to a specific maximum value, i.e., it causes horizontal EEG traces. This will interfere with frequency analysis. For our analyses we defined a rather conservative clipping setting by only considering a signal clipped, if it showed the horizontal line for longer than 10 
data points. We present the results from the (n)PSD analysis as DSA heatmaps.

\section{Results}

\subsection{Changes in the amplitude resolution for EEG display affect the stored EEG amplitude}

When calculating the PSD from the EEG recording our PSD resolution was $0.35 \mathrm{~Hz}$. The DSA in Fig. 1a shows a change in PSD with every change in the amplitude resolution display setting. The finer the amplitude resolution the higher the power, depicted by the warmer colors in Fig. 1a. We could correct for the differences in power by using the nPSD approach as displayed in Fig. 1b.

The analyses of the simulated EEG confirmed the influence of the amplitude setting on the display settings as presented in the DSA plot in Fig. 2a. The signal recorded from the SEDLine with the different amplitude settings during the $30 \mathrm{~mm} / \mathrm{s}$ setting replay shows the difference in recorded signal amplitude as well (Fig. 3). The jump in the DSA occurs because the sampling rate changes without being documented. If you then calculate the DSA with a set frequency resolution, i.e., a defined number of NFFT (number of discrete Fourier transformation) points like 512 in our case, the frequency resolution will be twice as fine in case you change from the initial sampling rate of 178 to $89 \mathrm{~Hz}$.

\subsection{Changes in the feed for EEG display affect sample rate of the stored EEG}

Further, the wider corridors of unchanged power starting at step 9, i.e., when we changed the feed of the EEG to $30 \mathrm{~mm} / \mathrm{s}$, indicate an influence of the feed setting on the sample rate. Another indicator is the change of the dominant oscillatory component around $22 \mathrm{~Hz}$ in steps 1 to 8 that got shifted to around $11 \mathrm{~Hz}$ in step 9 to 16 . When using the DSA derived from the nPSD, this shift is even more obvious. Because the recording was started with $30 \mathrm{~mm} / \mathrm{s}$ and changed to $15 \mathrm{~mm} / \mathrm{s}$ before starting the protocol, the dominant $\sim 10 \mathrm{~Hz}$ oscillatory activity observed in the beginning in a patient under general anesthesia/sedation was shifted to $\sim 20 \mathrm{~Hz}$. The change in sample rate is also reflected in an inaccuracy in calculating the length of the recording. When dividing the number of recorded data points per EEG channel by the indicated sample rate we found recording time of around $25 \mathrm{~min}$ which does not match the real recording time of around 33 min consisting of 16 steps a 2 min and sometime before and after the protocolled steps. When using the nPSD the jump of the power peak from $\sim 10$ to $\sim 20 \mathrm{~Hz}$ as a consequence of changing the feed from 30 to $15 \mathrm{~mm} / \mathrm{s}$ (Fig. 2b). Also the width of the $9-11 \mathrm{~Hz}$ frequency band with strong power in Fig. 2a and $\mathrm{b}$ becomes wider, because of the finer frequency resolution, due to the reduced sample rate but keeping the same Discrete Fourier Transformation settings applied by the pwelch command.

\subsection{EEG distortions: Clipping and low resolution}

Depending on the amplitude resolution the recorded EEG is differently affected by the clipping distortion. The higher the amplitude resolution on the display, the higher the amount of clipping as displayed in Fig. 1c. Especially the 1 and $2 \mu \mathrm{V} /$ $\mathrm{mm}$ settings can lead to a considerable amount of clipped EEG as detected with our 10-point setting for the clipping function. For the low amplitude resolution settings of 50 and $100 \mu \mathrm{V} / \mathrm{mm}$ we also observed horizontal EEG that was not caused by clipping, but by a very low resolution of the recorded EEG that led to a stair-like signal. Figure 4 presents $5 \mathrm{~s}$ examples of EEG raw traces (from the second 8 blocks).
Fig. 2 Density spectral arrays (DSA) of the (relative) power spectral density (PSD) for the different display settings depicted above the DSA for the simulated 9-11 Hz oscillatory activity. a DSA of the spectral power: Depending on the amplitude setting the recorded EEG amplitudes, i.e., the colors in the DSA change. b DSA indicating the relative power: The sudden jump in the dominant frequency range of $9-11 \mathrm{~Hz}$ because of the change in the feed from $30 \mathrm{~mm} / \mathrm{s}$ to $15 \mathrm{~mm} / \mathrm{s}$ is clearly identifiable

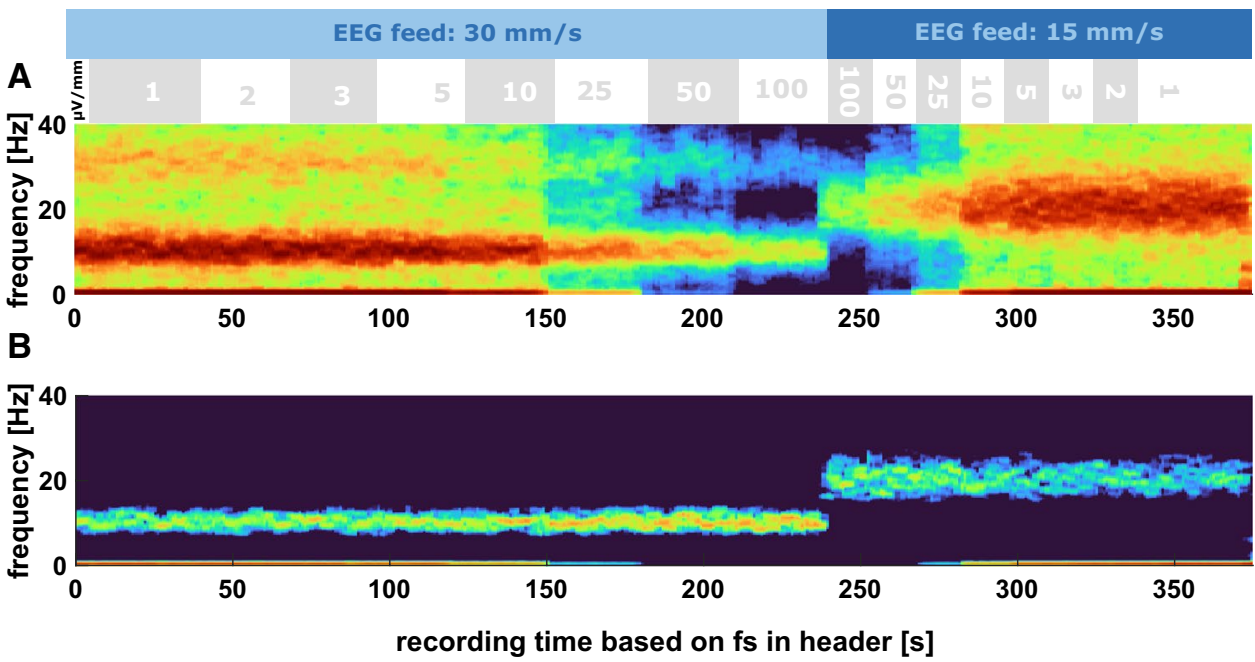


EEGL1Fp1
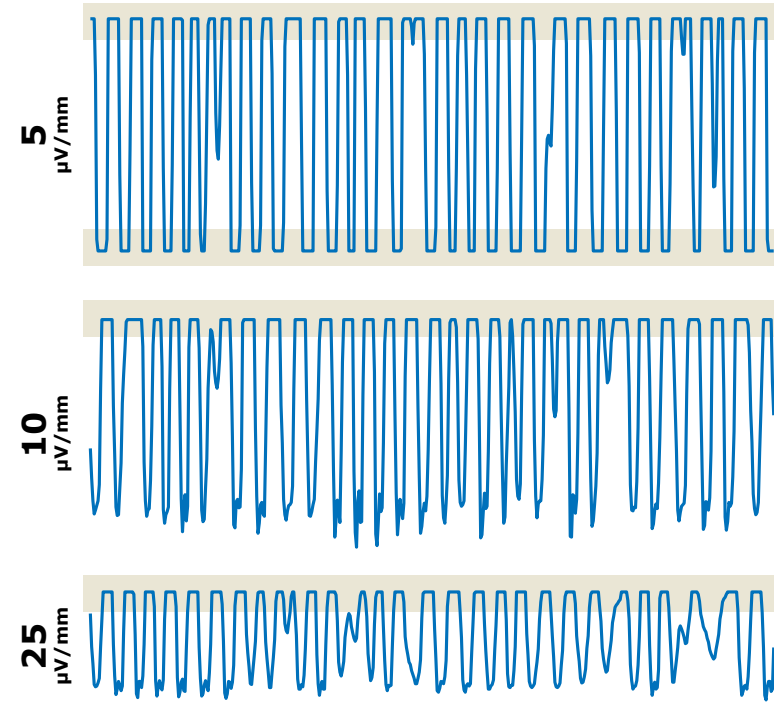

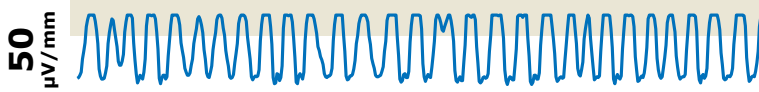

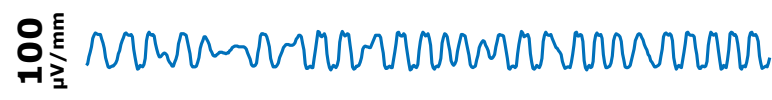
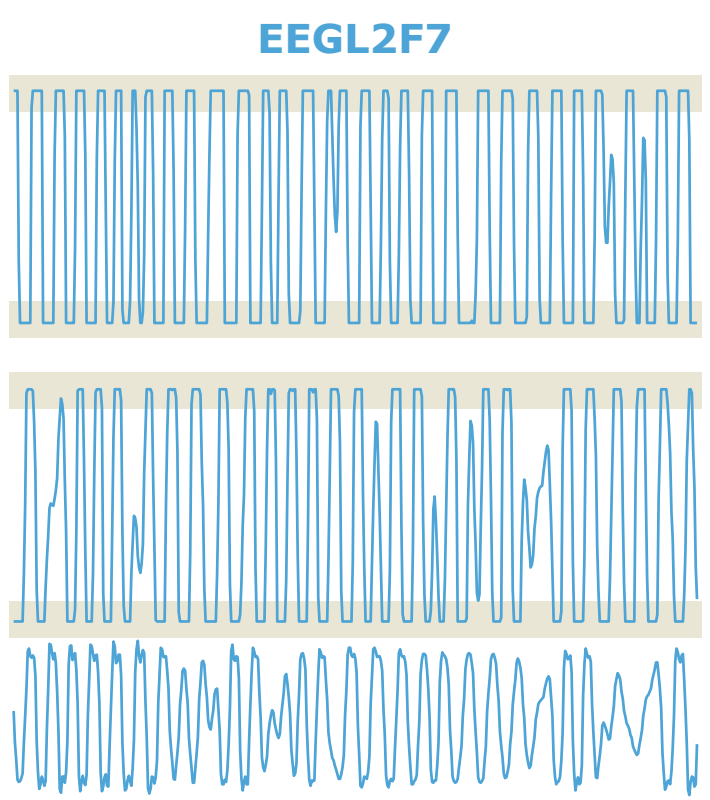

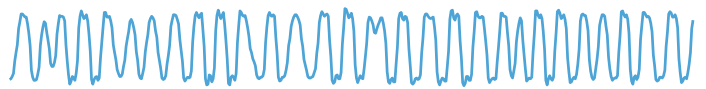

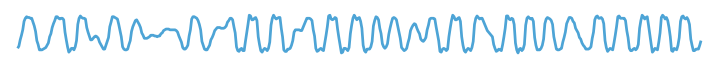

Fig. 3 Snippets of the recorded traces from the replay simulated 9-11 Hz oscillatory activity for the different amplitude settings on the display and for the different electrodes Fp1 (left) and F7 (right). The lower the resolution, the less the trace is affected by clipping. The

The number of possible amplitude values as depicted in Fig. 1d "number of state" graph strongly decreased when the amplitude resolution on the display was decreased, i.e. the signal starts to look "rectangular" as in Fig. 4d. But the amount of clipping seems to depend on the channel and the position of the signal on the screen. Figure 3 that displays the recorded simulated data at different amplitude resolutions revealed that in channel L1, clipping occurs also in the amplitude settings 25 and $50 \mu \mathrm{V} / \mathrm{mm}$, but it does not in channel L2. But in channel L2 we observed a lower-bound clipping in the $10 \mu \mathrm{V} / \mathrm{mm}$ setting. This means that the signal recorded reflects the signal displayed on the screen. L1 is displayed as first of the four EEG traces and is hence influenced by the upper-bound clipping. L2 is the third trace and at the $10 \mu \mathrm{V} / \mathrm{mm}$ the signal goes out of the display and hence becomes clipped. The screenshots in the supplemental Fig. S1 should clarify this issue. amount of clipping is dependent on the channel position in the display. Because the Fp1 trace is presented in the 1st row of the display this trace is affected by upper clipping for more settings than the F7 trace placed in the center of the SEDLine display

\section{Discussion}

Using the information provided by EEG recordings is recommended to monitor the patient when undergoing a surgical intervention requiring general anesthesia $[7,8]$. Processed EEG indices like the BiSpectral index [9] or the Patient State Index [2] attempt to estimate the hypnotic state of a patient as a dimensionless number. For a more reliable assessment of the patient's state, additional inspection of the raw EEG or the spectrogram is strongly suggested [10]. Further, in order to understand the processed EEG indices in more detail and to search for EEG features that can help to improve the reliability of these indices, the recording of intraoperative EEG is necessary. The SEDLine device allows for export of raw EEG via an USB port. This export function could provide a very useful tool to get EEG recordings to conduct research on. Unfortunately, as described by our results, the exported EEG recordings are dependent on the EEG setting for display on the monitor.

(A) A change in display feed causes a (undocumented) change in sample rate of the recorded EEG. 

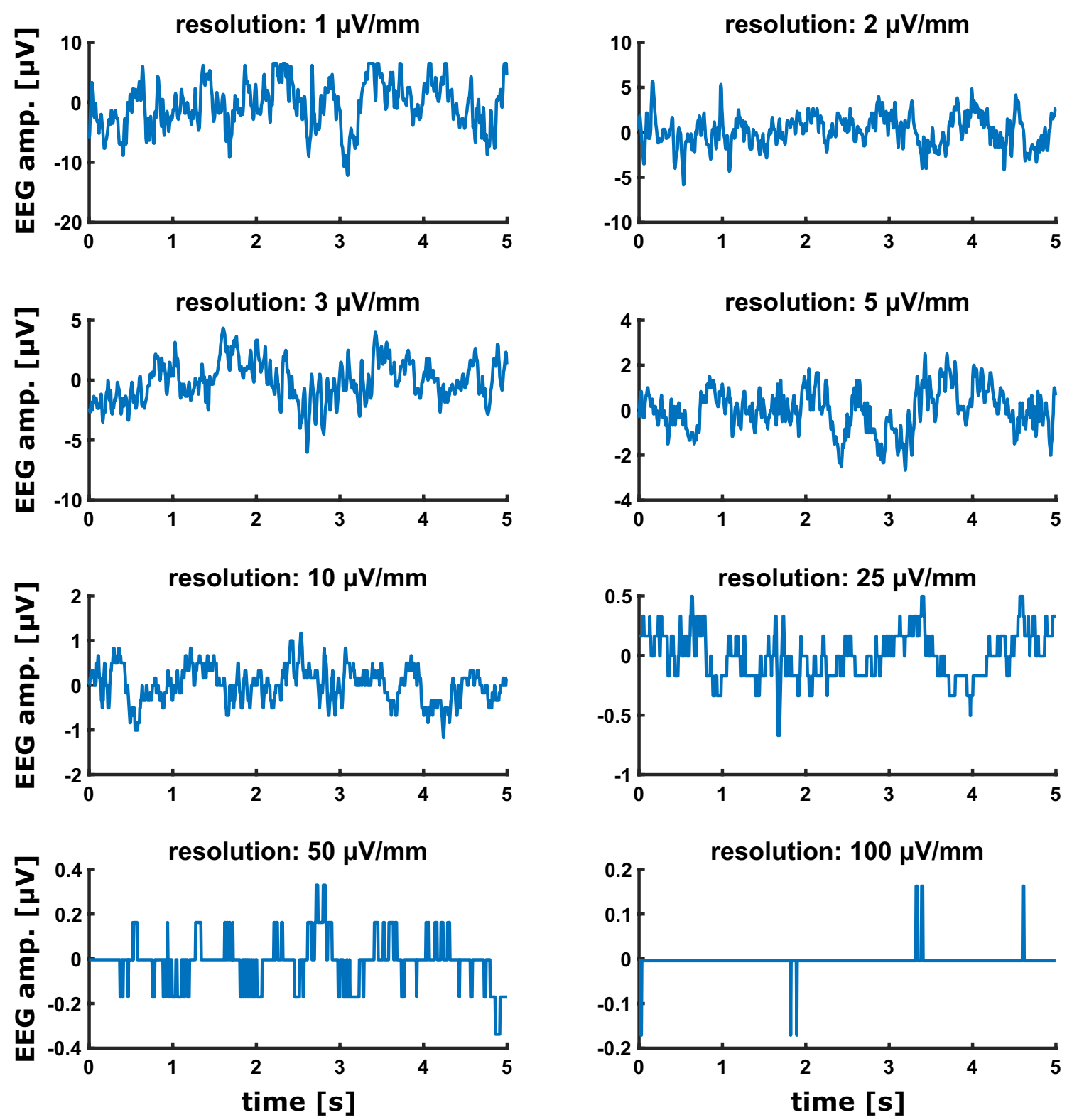

Fig. 4 Five second EEG samples recorded during the different EEG amplitude settings on the SEDLine display. The coarser the amplitude scaling on the display, the more stair-like the recorded EEG becomes

(B) A change in display amplitude resolution causes a (undocumented) change in the amplitude and affects the quantization of the recorded EEG.

(C) Inadequate amplitude resolution can lead to distortion of the EEG signal (clipping or stair-steps).

(D) The amount of clipping is dependent on the position of the displayed signal on the screen.

When the anesthesiologist starts a recording, the actual sample rate that is dependent on the EEG feed on the display is stored. This sample rate is then denoted in the header section of the EDF file after EEG export. Any changes made to the feed on the display will cause a change in the sample rate at which the EEG is recorded. However, these changes are not logged in the file. Hence, when analyzing the EEG from the EDF file, undocumented changes in the feed (sample rate) can lead to erroneous results. As displayed in Fig. 1 for patient data and Fig. 2 for simulated data, a change in the feed from 30 to $15 \mathrm{~mm} / \mathrm{s}$ causes a shift of the oscillatory alpha activity presented as a yellow to red band around $10 \mathrm{~Hz}$ in the spectrogram to a band around $20 \mathrm{~Hz}$. If the focus is on investigating alphaband properties, the result would be wrong, because the researcher would analyze the wrong frequencies. In order to check the raw EEG from the EDF file for these "change 
in feed" events, the duration of the recording should be checked for each case and compared to the proposed length of the recording, i.e., the number of data points in the EDF file divided by the sample rate denoted in the header. If these durations are off, a feed change probably happened. Further, the DSA can be checked. If there are jumps of a dominant band, like for instance alpha under general anesthesia, to either twice or half the frequency, a feed change can be assumed.

Another issue presents the change in the EEG amplitude as well as the quantization of the EEG when recorded at different amplitude resolutions on the display. A pure change in the EEG amplitude could be corrected, for instance, by normalizing the EEG amplitude by means of standard scoring, i.e., the presentation of the amplitude not as $(\mu) \mathrm{V}$ but as standard deviations, or as normalized PSD as presented in Figs. $1 \mathrm{~b}$ and $2 \mathrm{~b}$. But the choice of amplitude resolution also affects the quantization of the signal. The coarser the amplitude resolution on the display, the more stair-like the recorded EEG (Fig. 4) can be. This means that with lower display resolution, the number of recorded EEG amplitudes values can become lower. Lower quantization leads to an increased amount of white noise content in the spectrum [11]. For the spectral power of the EEG this means an increasing influence of the higher frequencies to the total power which is identifiable as warmer colors at $20 \mathrm{~Hz}$ and above in the 100 and $50 \mu \mathrm{V}$ settings for the patient EEG (Fig. 1b). While the quantization causes the reduction in the resolution and leads to an increased amount of zero-lines, the very fine resolution settings on the display like 1 or $2 \mu \mathrm{V} /$ $\mathrm{mm}$ for the patient EEG or from $1 \mu \mathrm{V} / \mathrm{mm}$ to $10 \mu \mathrm{V} / \mathrm{mm}$ (or even $25 \mu \mathrm{V} / \mathrm{mm}$ in the Fp1 channel) for the simulated data can lead to another problem: clipping. EEG clipping occurs if the amplitude goes into saturation and the maximal (or minimal) possible amplitude values are reached. In the case of the SEDLine we observed hard clipping, i.e., a horizontal line in the time series once the EEG amplitude reached the threshold. Normally, with well-adjusted amplifier settings, clipping occurs during large amplitude artifacts with amplitudes considerably larger than the useful signal like the EEG. In our case presented, clipping affected the artifact-free EEG. Clipped signals cannot be completely recovered, although by assuming an underlying model structure the missing parts can be estimated [12]. Hence, clipping can affect the analytical results. For instance, the (power) spectrum changes with clipping [13]. In order to avoid the quantization as well as the clipping issue the 3 and $5 \mu \mathrm{V} /$ $\mathrm{mm}$ display setting were best suited in the presented EEG measurement. But the 3 and $5 \mu \mathrm{V} / \mathrm{mm}$ setting would not work for the simulated data because of strong clipping. So the display has to be carefully adjusted and each change, if necessary, has to be noted.
In summary, we here investigated and described the impact of the SEDLine display settings on the EEG traces that can be extracted from the USB port of the device in the EDF format. The recorded EEG presents the traces as they are displayed on the screen. If the SEDLine is used for collecting EEG that will be analyzed for scientific questions the display settings should not be changed throughout data collection. Further, the amplitude resolution on the display should be set to a factor that allows collection of clipping free data at a high resolution.

Acknowledgements The authors would like to thank Fabian Nowak for the great help with the EEG clipping detection

Author contributions FvD helped design the experiments, analyze the data, discuss the results, and write the manuscript. CJ helped design the experiments, collect the data, analyze the data, and write the manuscript. GS helped discuss the results and write the manuscript. PG helped design the experiments, discuss the results, and write the manuscript. MK helped design the experiments, analyze the data, discuss the results, and write the manuscript.

Funding Open Access funding provided by Projekt DEAL.

\section{Compliance with ethical standards}

Conflict of interest The authors declare that they have no conflict of interest.

Ethical approval This study was performed in accordance with the ethical standards as laid down in the 1964 Declaration of Helsinki and its later amendments. It was approved and the requirement for written informed consent was waived by the Institutional Research Ethics Committee of Charité - Universitätsmedizin Berlin (vote number: EA1/151/16)

Open Access This article is licensed under a Creative Commons Attribution 4.0 International License, which permits use, sharing, adaptation, distribution and reproduction in any medium or format, as long as you give appropriate credit to the original author(s) and the source, provide a link to the Creative Commons licence, and indicate if changes were made. The images or other third party material in this article are included in the article's Creative Commons licence, unless indicated otherwise in a credit line to the material. If material is not included in the article's Creative Commons licence and your intended use is not permitted by statutory regulation or exceeds the permitted use, you will need to obtain permission directly from the copyright holder. To view a copy of this licence, visit http://creativecommons.org/licenses/by/4.0/.

\section{References}

1. Brown EN, Lydic R, Schiff ND. General anesthesia, sleep, and coma. N Engl J Med. 2010;363(27):2638-50. https://doi. org/10.1056/NEJMra0808281.

2. Prichep L, Gugino L, John E, Chabot R, Howard B, Merkin H, Tom M, Wolter S, Rausch L, Kox W. The Patient State Index as an indicator of the level of hypnosis under general anaesthesia. $\mathrm{Br}$ J Anaesth. 2004;92(3):393-9. 
3. Kemp B, Värri A, Rosa AC, Nielsen KD, Gade J. A simple format for exchange of digitized polygraphic recordings. Electroencephalogr Clin Neurophysiol. 1992;82(5):391-3. https://doi. org/10.1016/0013-4694(92)90009-7.

4. Hesse S, Kreuzer M, Hight D, Gaskell A, Devari P, Singh D, Taylor N, Whalin M, Lee S, Sleigh J. Association of electroencephalogram trajectories during emergence from anaesthesia with delirium in the post-anaesthesia care unit: an early sign of postoperative complications. Br J Anaesth. 2019;122(5):622-34.

5. Akeju O, Westover MB, Pavone KJ, Sampson AL, Hartnack KE, Brown EN, Purdon PL. Effects of sevoflurane and propofol on frontal electroencephalogram power and coherence. Anesthesiology. 2014;121(5):990-8.

6. Kreuzer M, Kochs EF, Pilge S, Stockmanns G, Schneider G. Construction of the Electroencephalogram Player: A Device to Present Electroencephalogram Data to Electroencephalogram-Based Anesthesia Monitors. Anesth Analg. 2007;104(1):135-9.

7. Aldecoa C, Bettelli G, Bilotta F, Sanders RD, Audisio R, Borozdina A, Cherubini A, Jones C, Kehlet H, MacLullich A. European Society of Anaesthesiology evidence-based and consensus-based guideline on postoperative delirium. Eur J Anaesthesiol. 2017;34(4):192-214.

8. Chan MTV, Hedrick TL, Egan TD, García PS, Koch S, Purdon PL, Ramsay MA, Miller TE, McEvoy MD, Gan TJ. American Society for Enhanced Recovery and Perioperative Quality Initiative Joint Consensus Statement on the Role of Neuromonitoring in Perioperative Outcomes: Electroencephalography. Anesth Analg.
2020;130(5):1278-91. https://doi.org/10.1213/ane.0000000000 004502.

9. Rampil IJ. A primer for EEG signal processing in anesthesia. Anesthesiology. 1998;89:980-1002.

10. Herman ST, Abend NS, Bleck TP, Chapman KE, Drislane FW, Emerson RG, Gerard EE, Hahn CD, Husain AM, Kaplan PW, LaRoche SM, Nuwer MR, Quigg M, Riviello JJ, Schmitt SE, Simmons LA, Tsuchida TN, Hirsch LJ, Critical Care Continuous EEGTFotACNS. Consensus statement on continuous EEG in critically ill adults and children, part II: personnel, technical specifications, and clinical practice. J Clin Neurophysiol. 2015;32(2):96-108. https://doi.org/10.1097/WNP.0000000000 000165 .

11. Bennett WR. Spectra of quantized signals.\&nbsp;The Bell System . Tech J. 1948;27(3):446-72.

12. Weinstein AJ, Wakin MB. (2011) Recovering a clipped signal in sparseland. arXiv preprint arXiv: 11105063.

13. Levitt H, Cudahy E, Hwang WH, Kennedy E, Link C. Towards a general measure of distortion. J Rehabil Res Dev. 1987;24(4):283-92.

Publisher's Note Springer Nature remains neutral with regard to jurisdictional claims in published maps and institutional affiliations. 